\title{
Comparison Performance of Efficient Adaptive Temporal Filters and Spatial Arrays Antennas based on Fractional Lower-Order Statistics in non-Gaussian Environment
}

\author{
E. Del Re, and M. Rupi. \\ LENST Laboratory - Department of Electronic Engineering \\ Facoltà di Ingegneria, Università di Firenze \\ Via di Santa Marta 3, 50139 FIRENZE, Italy \\ $\mathrm{Tel} / \mathrm{Fax}+390554796271 /+390554796485$
}

\begin{abstract}
Adaptive channel equalization is an effective tool for the antenna receiver for estimating the information sequence in severe interference backgrounds. As a result, the problem of linear channel distortion or multipath suppression has been the focus of considerable research in the signal processing and communications communities.

The choice of the antenna receiver structure is a challenge for the next radio communication systems, because from this aspect will depend great part of the improvement it is possible to obtain with efficient dsp devices. The two possible streets are the use of omnidirectional antennas or the introduction of antennas array.

In our paper we evaluate a comparison between these two different choice considering adaptive equalizer in different environmental conditions. Basically, we studied and proposed new solutions for dealing with good Gaussian and bad nonGaussian background noise.
\end{abstract}

\section{Introduction}

The Constant Modulus Algorithm (CMA) was studied by Treichler and Larimore [7] who analyzed its performance in terms of capture and lock behavior. Initial CMA studies considered only the temporal diversity at the receiver. The development of advanced division access techniques has made the concept of spatial diversity worth-pursuing. As a consequence, directional array antenna beamformers have taken the place of omnidirectional antennas. In this context, Gooch and Lundel introduced the so-called constant modulus array, which exploits the constant modulus properties of the communication signal of interest to steer a beam in the direction of the information sequence while placing nulls in the directions of interferences [1].

Most of the theoretical work on blind equalizers based on the CM criterion typically exploit higher (than second) order statistics or second-order cyclostationary statistics of the channel output signal. For this reason these methods have focused on the case where the channel noise is assumed to follow the Gaussian model. The Gaussian assumption is frequently motivated because it often leads to mathematically tractable solutions. However, algorithms designed under the Gaussian assumption exhibit various degrees of performance degradation, depending on the non-Gaussian nature of the environment. For some applications, such degradation is so strong to compromise irreparably the information transmitted over such impulsive channels. In these cases, there is a point break after which is not possible to recover the desired signal. As we proved in the simulation experiments, for the Constant Modulus Algorithm class, the presence of heavy-tail nature noise, even though not much impulsive, (i.e. realistic near Gaussian values), lead to a total lost of performance if used with methods optima under the Gaussianity assumption.

Indeed, experimental results have been reported where electromagnetic noise in urban mobile-radio channels is heavy-tailed in nature and cannot be modeled by means of Gaussian or other exponentialtailed distributions [2, 3]. In addition, impulsive channels appear in telephone lines, underwater acoustic communications (ice-cracks), atmospheric environments (thunderstorms), and mobile communications.

Hence, there is a need to use more general and realistic non-Gaussian models and design efficient equalization techniques that take into account the possible heavy-tail nature of the data, and simulta- 
neously work well in good Gaussian channels.

Our work is devoted to the development of a novel constant modulus method which makes use of temporal filter diversity and array signal processing system for robust performance in the presence of interference/noise environments that can be modeled according to the alpha-stable law. We compare the performance of a temporal FIR filter and an antenna array model with complex coefficients which both use a fractional lower-order statistics on computing the adaptation iterative law in presence of impulsive noise and Gaussian channels. Performance is expressed in terms of bit error rate (BER), which has been derived by simulations under the assumption of correlated signals.

\section{Alpha Stable Distributions}

Man-made as well as natural physical processes can generate interferences containing noise components that are impulsive in nature. In modeling this type of signals the symmetric alpha-stable $(S \alpha S)$ distribution provides an attractive theoretical tool. It was proven that under broad conditions, a general class of heavy-tailed noise follows the stable law [4].

The $S \alpha S$ class of distributions is best defined by its characteristic function, depending by three parameters. The characteristic exponent $\alpha$ is the most important parameter of the $S \alpha S$ distribution and it determines the shape of the distribution. The smaller the characteristic exponent $\alpha$ is, the heavier the tails of the alpha-stable density. It is this heavy-tail characteristic that makes the alpha-stable densities appropriate for modeling noise that may be impulsive in nature. We should also note that the stable distribution corresponding to $\alpha=2$ coincides with the Gaussian density. The dispersion parameter $\gamma$ determines the spread of the distribution around its location parameter $\delta$ (third and last parameter), much in the same way that the variance of the Gaussian distribution determines the spread around the mean.

The appeal of $S \alpha S$ distributions as statistical models derives from some important properties. They: (i) naturally arise as limiting processes via the Generalized Central Limit Theorem; (ii) possess the stability property and share many features with the Gaussian density such as unimodality, symmetry with respect to the location parameter, bell-shape; (iii) all but Gaussian distributions possess finite moments of order $p$ only when $p$ is strictly less than $\alpha: E|X|^{p}<\infty$ for $p<\alpha$. Strictly related with this last property, the main concept to point out for this class of distribution is the introduction of fractional-lower order moments as the only possible tool capable to digitally process the analyzing data.

\section{Constant Modulus Methods}

Let suppose that the received signal $x(n)$ is applied to a transversal (tapped delay line) FIR filter with adjustable complex coefficients. We may write the complex filter output as

$$
y(n)=\mathbf{x}^{t}(n) \mathbf{w}(n)
$$

where $\mathbf{x}(n)$ is the vector of data in the filter delay line, $\mathbf{x}(n)=[x(n) x(n-1) \cdots x(n-$ $N+1)]^{T}$ and $\mathbf{w}(n)$ is the $N \times 1$ vector of adjustable coefficients. If we consider an uniform linear array system, with equispaced antennas of $d$ and wavelength $\lambda$, the received spatial vector is $\mathbf{x}(n)=\left[x(n) e^{-j \omega_{1}}, x(n) e^{-j \omega_{2}}, \cdots, x(n) e^{-j \omega_{N}}\right]^{T}$, where $\omega_{i}=2 \pi d / \lambda \sin \left(\theta_{i}\right)$, and $\theta_{i}$ is the direction of arrival evaluated respect with the orthogonal to the antenna.

The goal in this adaptive filtering is to restore $y(n)$ to a form near as possible to the original version of the transmitted sequence, i.e. to a signal which, on the average, has a constant instantaneous modulus. This may be done by choosing the coefficient vector $\mathbf{w}(n)$ in such a way as to minimize a positive definite measure of the signal modulus variation. The constant modulus family of blind equalizers is based on a cost function that assigns a penalty to deviations in the modulus of the controller's complex output signal. The cost function is given by

$$
J_{p q}^{C M}=E\left[\|\| y(n) \|^{p}-\left.\delta\right|^{q}\right]
$$

where $E[\cdot]$ denotes statistical expectation, $p$ and $q$ are positive integers, and $\delta$ is a constant greater than zero, strictly related to the constant modulus.

The most famous member of this family is the Constant Modulus Algorithm (CMA) for which both parameters $p$ and $q$ are equal to two [5, 6]. The CMA attempts to minimize the cost function shown in (2) by following the path of steepest descent. The update equation for the $p=2$ and $q=2$ case is given by

$$
\begin{aligned}
\mathbf{w}(n+1) & =\mathbf{w}(n)-\mu \mathbf{x}^{*}(n) \epsilon(n) \\
\epsilon(n) & =y(n)\|y(n)\|^{2}-y(n)
\end{aligned}
$$

The main characteristic associated with the standard CMA is that it involves fourth-order moments 
of the signal, as the cost function in (2) points out. In the presence of heavy-tailed noise, the use of secondor higher-order statistics in effect amplifies the noise.

\section{Temporal and Spatial FLOS CM}

The physical phenomena which appear to involve dramatically the quality of a good reception of the signal during a transmission are one of the most critical aspect in a radio communication scenario. That is why a good representation of such complex variety is became the goal of new and innovative systems modeling. Toward such destination, our efforts have tried to simulate a more realistic environment in which the noise additive background statistics were of impulsive nature. However, this hypothesis is not restrictive and moreover includes the most popular and commonly used Gaussian statistic.

For such cases, we propose a new cost function that points out mainly two aspects. First, we are processing signals with constant modulus modulation, so this characteristic is what we eventually desire; second, we suppose to have to deal with a larger and more realistic class of noise distributions, which may give origin to some impulsiveness totally disruptive if managed with the standard CMA. What we present, is a modified version of the error function which takes into account this heavy-tailed nature of the noise, trying to mitigate its impulsiveness by means of fractional lower-order statistics (FLOS). The new cost function has the expression

$$
J_{p, q}^{F L O S-C M}=E\left[||\left|y(n) \|^{(p-1)} y(n)-\delta y(n)\right|^{q}\right]
$$

where $\delta$ is a $p$ th -1 power version of the constant signal modulus, assuming $p<\alpha$. The pair $(p, q)$ takes values, possibly fractional, between 0 and $\alpha$, where $\alpha$ is the characteristic exponent of the alpha-stable distribution that best describes the statistics of the noise vector $\mathbf{n}(t)$. Furthermore, the product $p \cdot q$ must be less than $\alpha$.

The equations we came out is

$$
\begin{aligned}
& \mathbf{w}(n+1)=\mathbf{w}(n)-\mu \nabla_{w^{*}} J_{p q}= \\
& \mathbf{w}(n)-\mu \frac{q}{2}\|\bar{A}\|^{q-2} \mathbf{x}(n)^{*}\left(p\|y(n)\|^{p-1}-1\right) \bar{A} \\
& \bar{A}=y(n)\left(\|y(n)\|^{p-1}-1\right)
\end{aligned}
$$

Similar studies can be carried out for the temporal and spatial analysis in case of sinusoid signals. Our approach permit to opportunely initialize the weights vector in order to have a priori knowledge of the algorithm in terms of lock (desired signal) and capture (interference signal) behavior. One difference between the two parallel analysis is the capability to discriminate this two lock and capture zones for the temporal system only if the incoming signals are tones. Adversely, in the case of antennas array it is always possible to find a more efficient expression due to the presence of another information term carried out with the signals represented by the angular spatial arrival direction. This is an important point which can explain the diversity in the bit error rate curves obtained during the simulation experiments.

\section{Simulations Results}

In this section, we test and validate the new FLOSCM temporal-spatial adaptive algorithms and compare their performance with that of the conventional $\mathrm{CM}$ algorithms in a noisy environment.

For the spatial model, let consider two independent transmitted signals, QPSK modulated, impinging on the array of dimension two from directions $\theta=\left[30^{\circ},-40^{\circ}\right]$. The desired signal is supposed to be the first signal coming from $30^{\circ} \mathrm{deg}$ direction.

For the temporal model, let suppose a filter length of two taps, and consider a received signal which is a scalar sum of the direct signal and a delayed version of itself. A power control system is supposed working at the receiver, as has been standardized for the next generation systems.

The number of snapshots available to the array is $M=100,000, M=500,000$ or $M=1,000,000$ depending on the expected BER and we performed 10 Montecarlo runs.

In the Figures 1 and 2 we plot the Bit Error Rate versus the SNR for the original CM and the proposed FLOS-CM algorithms for both the filter and the array solutions. In Figure 1 the noise component is modeled as an alpha-stable process with $\alpha=1.85$ (i.e., the noise is fairly close to Gaussian, truly measured for some particular communication transmissions). Figure 1 demonstrate that occurrences of noise outliers during the adaptation, have an adverse affect to the learning curve of the original CM method. In fact, even a relative low impulsiveness of $\alpha=1.85$ has a disruptive effect on the convergence behavior of the classical CM algorithms. Briefly speaking, as an impulse occurs, the classical CM algorithm looses its convergence properties and it becomes not able anymore to recover the transmitted information se- 
quence. In this case we can see the practical horizontal upper curve of BER in the graph. On the other hand, the proposed FLOS-CM Filter and FLOS-CM Array cost function can suppress the noise components and results in a much deeper bit error rate curve. However, as it is possible to see from the two lower curves in the Figure 1, the spatial solution is more robust respect with the temporal ones. The gain that we can obtain is almost $10 \mathrm{~dB}$ for low SNR and $2-3 \mathrm{~dB}$ for higher SNR.

As aforementioned, the difference between the two performance behavior is due to the capability for the array system to opportunely initialize the algorithm in order to obtain a more stable and faster convergence. The possibility to discriminate the incoming signals with the arrival angles allows the array to have a further information which is the focus of such analysis.

In Figure 2 the noise component is modeled as a Gaussian random variable process. In the presence of Gaussian channels, the CM performs better than the FLOS-CM for both the spatial and the temporal models, as we could expect. In fact, in presence of Gaussian noise, the CM algorithm gives the optimum solution, since does not use any fractional power, for which it is always necessary an approximation to deal with.

Eventually, if we compare the two Figures 1 and 2, we can extract an important result. For a wide range of SNR values, between 4 and $14 \mathrm{~dB}$ for the filter analysis and between 4 and $8 \mathrm{~dB}$ for the array system, the FLOS-CM with a relatively low value of impulsiveness $(\alpha=1.85)$ has comparable performance with the CM in Gaussian channels, as the curves show. This testifies a very stable behavior of the proposed algorithm over different noise conditions.

\section{Conclusions}

We proposed a new method for blind equalization of communication signals using a constant modulus criterion based on fractional lower-order statistics.

The introduced FLOS-CM algorithms for a filter and an array of sensors exploits the constant modulus property of the signal of interest and uses the heavy-tailed noise suppression capabilities of FLOS to compensate the fading and multipath effects due to a real radio communication transmission in order to attenuate the influence of the interference and noise. The main advantage of the proposed method is its robustness in the presence of various noise environ-

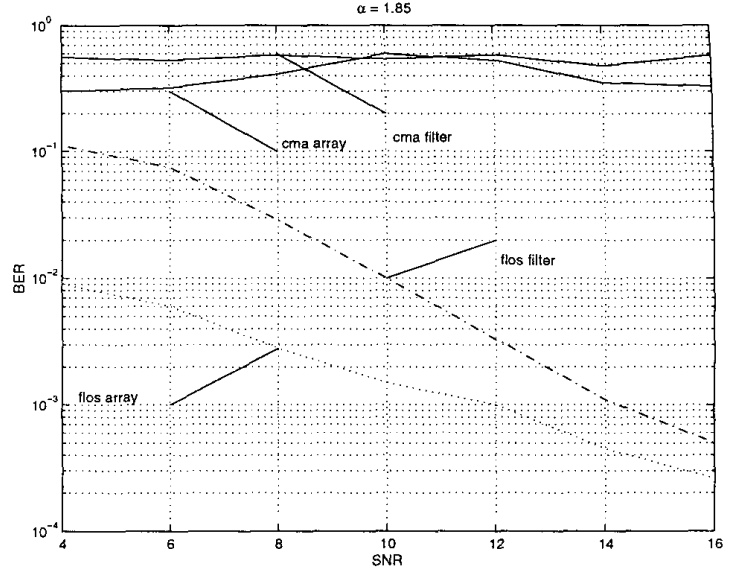

Figure 1: Bit error rate curves for the filter receiver system in configuration for $\alpha=1.85$ and $\alpha=2$.

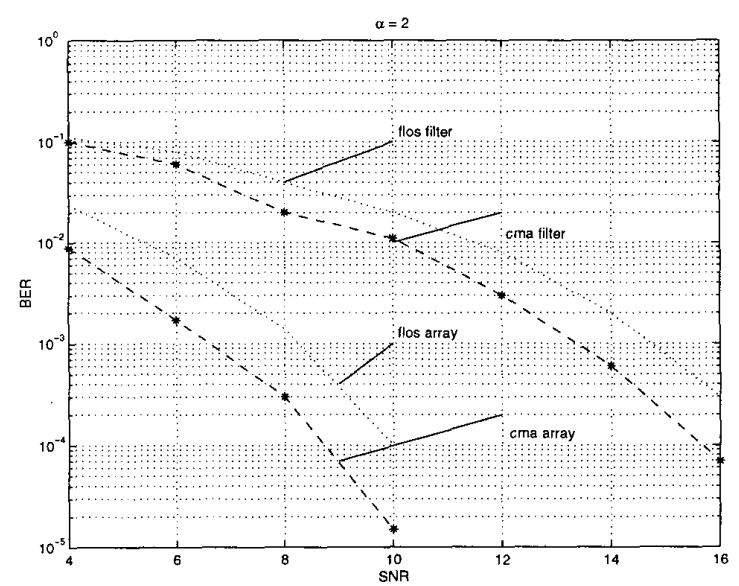

Figure 2: Bit error rate curves for the filter receiver system in configuration for $\alpha=1.85$ and $\alpha=2$.

ments.

The capability of an array system to steer a beam in the direction of the signal while suppressing interference and noise is resulting in a better performance in terms of bit error rate respect to the equivalent FIR temporal filter case.

\section{References}

[1] R. Gooch and J. Lundell. The cm array: an adaptive beamformer for constant modulus signals. In Proceedings of International Conference on Acoustic, speech, and Signal Processing, 1986.

[2] D. Middleton. Statistical-phisical models of urban radio noise environment - part i: Founda- 
tions. IEEE Transections Elect. Comput., vol 14, pp 38-56, 1972.

[3] D. Mitra and H.V. Poor. Detection of spreadspectrum signals in multi-user environment. In Proc. of IEEE ICASSP, pp 1844-I847, 1995.

[4] C L Nikias and M Shao. Signal Processing with Alpha-Stable Distributions and Applications. John Wiley and Sons, New York, 1995.

[5] J.R. Treichler and B.G. Agee. A new approach to multipath correction of constant modulus signals. IEEE Transactions on Acoustic, speech, and Signal Processing, Apr 1983.

[6] J.R. Treichler and M.G. Larimore. New processing techniques based on the constant modulus adaptive algorithm. IEEE Transactions on Acoustic, Speech, and Signal Processing, Apr 1985.

[7] J.R. Treichler and M.G. Larimore. The tone capture properties of cma-based interference suppressors. IEEE Transactions on Acoustic, Speech, and Signal Processing, Aug 1985. 